ISSN 0103-5150

Fisioter. Mov., Curitiba, v. 25, n. 2, p. 399-409, abr./jun. 2012 Licenciado sob uma Licença Creative Commons

\title{
Efeito do treinamento combinado e aeróbio no controle glicêmico no diabetes tipo 2
}

\author{
Effect of combined and aerobic training on \\ glycemic control in type 2 diabetes
}

\section{Antônio Renato Pereira Moro ${ }^{[a]}$, Rodrigo da Rosa Iop ${ }^{[b]}$, Franciele Cascaes da Silva ${ }^{[c]}$, Paulo José Barbosa Gutierres Filho ${ }^{[\mathrm{d}]}$}

[a] Educador Físico, Doutor em Ciência do Movimento Humano, docente do Departamento de Educação Física da Universidade Federal de Santa Catarina (CDS/UFSC), Florianópolis, SC - Brasil, e-mail: moro@cds.ufsc.br

[b] Mestre em Ciências Aplicadas Atividade Física e Saúde, docente do Departamento de Fisioterapia da Universidade do Sul de Santa Catarina (UNISUL), Tubarão, SC - Brasil, e-mail: fisioptb@hotmail.com.

[c] Fisioterapeuta, bolsista do curso de Fisioterapia pela Universidade do Sul de Santa Catarina (UNISUL), Tubarão, SC Brasil, e-mail: francascaes@yahoo.com.br

[d] Doutor, professor de Educação Física, pesquisador no Laboratório de Atividade Motora Adaptada (LABAMA), Universidade do Estado de Santa Catarina (UDESC), Florianópolis, SC - Brasil, e-mail: d2paulo@udesc.br

\section{Resumo}

Introdução: 0 diabetes tipo 2 é um grupo heterogêneo de doença metabólica causada por uma disfunção na secreção da insulina e/ou ação desta. Objetivos: Comparar o efeito de duas modalidades de treinamento, o combinado (aeróbio e resistido) e o aeróbio, no controle glicêmico no diabetes tipo 2. Materiais e métodos: A pesquisa caracteriza-se por ser um estudo quase-experimental. Após aprovação do CEP, com registro 09.071.4.08. III, deu-se início ao programa de treinamento combinado e aeróbio. Foram selecionados 24 participantes, de ambos os gêneros, sedentários, com média de idade de 60,41 \pm 7,87. Os participantes foram divididos aleatoriamente em dois grupos: treinamento combinado $(n=12)$ e treinamento aeróbio ( $n$ =12); ambos foram avaliados no início e final do estudo. A concentração sérica de glicose foi determinada pelo sistema Vitros e a hemoglobina glicosilada foi determinada pelo método Cromatografia Líquida de Alta Performance. 0 treinamento foi realizado três vezes por semana, com duração total de 20 semanas. Os dados são expressos em média e desvio-padrão. Foi aplicado o teste t pareado $(p \leq 0,05)$ para comparar a média basal e após 20 semanas de treinamento. Resultados: A média da glicose em jejum do treinamento 
combinado reduziu significativamente, de 167,41 $\pm 38,13$ para $119,83 \pm 20,91$, sendo que o mesmo ocorreu com o treinamento aeróbio de $189,83 \pm 63,57$ para $139,91 \pm 34,04$. Os valores da hemoglobina glicosilada no treinamento combinado e treinamento aeróbio reduziram significativamente, de 8,61 $\pm 1,17$ para 7,25 $\pm 1,24$ e de $9,52 \pm 2,46$ para 8,37 $\pm 1,50$, respectivamente. Conclusão: 0 treinamento combinado foi mais eficaz em relação à hemoglobina glicosilada e o treinamento aeróbio, na glicose plasmática.

Palavras-chave: Diabetes tipo 2. Hemoglobina glicosilada. Glicose plasmática.

\begin{abstract}
Introduction: Type 2 diabetes is a heterogeneous group of metabolic disease caused by a dysfunction in insulin secretion and/or its action. Objectives: To compare the effect of two types of training, combined (aerobic and resistance) and aerobic exercise training, on glycemic control of type 2 diabetes. Materials and methods: The research is characterized by a quasi-experimental study. After approval of the research ethics commitee with record 09.071.4.08. III, it was started a program of combined and aerobic training. We selected 24 participants from both genders, sedentary, with a mean age of 60,41 $\pm 7,87$. Participants were randomly divided into two groups: combined training $(n=12)$ and aerobic training $(n=12)$. They were both evaluated at baseline and study end. Serum glucose was determined by the Vitros and glycosylated hemoglobin was determined by High Performance Liquid Chromatography. Training was carried out three times a week, in a total of 20 weeks. Data are expressed as mean and standard deviation. We used the paired test ( $p \leq 0.05)$ to compare the mean baseline and after 20 weeks of training. Results: The mean fasting glucose in combined training significantly reduced, from $167.41 \pm 38.13$ to $119.83 \pm 20.91$, and the same occurred with aerobic training, from $189.83 \pm$ 63.57 to $139.91 \pm 34.04$. The values of glycosylated hemoglobin in combined and aerobic training significantly reduced, from $8.61 \pm 1.17$ to $7.25 \pm 1.24$ and from $9.52 \pm 2.46$ to $8.37 \pm 1.50$, respectively. Conclusion: The combined training was more effective for glycosylated hemoglobin and aerobic training, on plasma glucose.
\end{abstract}

Keywords: Type 2 diabetes. Glycosylated hemoglobin. Blood glucose.

\section{Introdução}

O Diabetes tipo 2 (DT2) é definido pela Associação Americana de Diabetes (1) como um grupo heterogêneo de doenças metabólicas caracterizadas pela hiperglicemia, causada por um dos defeitos na secreção da insulina ou na ação desta, ou por ambas as coisas.

Segundo a Sociedade Brasileira de Diabetes (2) e a Sociedade Portuguesa de Diabetes (3), no DT2 ocorrem alterações no funcionamento endócrino que atingem principalmente o metabolismo dos carboidratos. A insulina interfere na manutenção do controle glicêmico, atuando na redução e manutenção a níveis considerados normais, mas também age no metabolismo das proteínas e lipídios. Além da ação hipoglicemiante, a insulina participa da lipogênese e da proteogênese, sendo o principal hormônio anabólico.

Existe uma epidemia de diabetes em curso. Em 1985, eram 30 milhões de pacientes no mundo todo; uma década mais tarde, o número triplicou (135 milhões). De acordo com a World Health Organization -
WHO (4), em 2000, existiam 177 milhões, mas esse quadro deverá aumentar para 370 milhões até 2030, aumentando a prevalência de 2,8\% em 2000 para $4,4 \%$.

Os dez principais países do mundo com maior prevalência de diabéticos são: Índia, China, Estados Unidos, Indonésia, Japão, Paquistão, Rússia, Brasil, Itália e Emirados Árabes Unidos (5).

No Brasil, calcula-se que, em 2025, possam existir cerca de 11 milhões de diabéticos no país, o que representa um aumento de mais de $100 \%$ em relação aos atuais 5 milhões de diabéticos, em 2000 (6).

Os custos de atenção ao diabetes variam de 2,5\% a 15\% dos orçamentos anuais da saúde, dependendo da prevalência local de diabetes e do nível de complexidade dos tratamentos disponíveis. Os custos de produção perdidos podem exceder, em até cinco vezes, os custos diretos de atenção à saúde, conforme estimativas obtidas em 25 países latino-americanos (5).

Estudos baseados em populações realizados na China, Canadá, Estados Unidos e em vários países 
europeus indicam que intervenções para alterar o estilo de vida no mundo podem prevenir o início do diabetes em pessoas de alto risco (5).

Um controle glicêmico inadequado pode acelerar o aparecimento de doenças cardiovasculares, renais e oftálmicas, resultando em risco elevado de morbidade e mortalidade (7).

As diretrizes atuais da American Diabetes Association, European Association Diabetes e American Heart Association recomendam exercícios aeróbio e resistido regular para pessoas com diabetes tipo $2 \mathrm{sem}$ maiores complicações $(8,9)$. No entanto, as diretrizes são escritas de maneira geral e não fornecem informações sobre a intensidade ou o tipo de treinamento mais benéfico em pessoas com diabetes (9-11).

$\mathrm{Na}$ escolha de um programa de exercícios para pessoas com DT2, deve-se levar em consideração o impacto do exercício no controle metabólico. Tradicionalmente, os programas de exercício para diabetes tipo 2 envolvem o regime de treinamento aeróbio, consistindo de exercício repetitivo de grandes grupos musculares que visam à aptidão cardiorrespiratória.

Segundo Zanuso et al. (11), o treinamento físico aeróbio melhora o controle glicêmico, a sensibilidade à insulina e o $\mathrm{VO}_{2 \text { máx }}$. Além disso, quanto mais intenso for exercício aeróbio, melhor será o controle glicêmico e a sensibilidade à insulina $(12,13)$.

Pesquisas recentes demonstraram os benefícios do treinamento resistido no DT2. No estudo de Cauza et al. (14), constataram que o treinamento de força foi mais eficaz que o treinamento aeróbio na melhora do controle glicêmico e no perfil lipídico.

Outros estudos, no entanto, relataram que o exercício combinado (aeróbio e resistido) pode ser ainda mais eficaz na redução da hemoglobina glicosilada (HbA1c) e na melhora da sensibilidade à insulina (15).

O controle glicêmico tem sido tradicionalmente 0 principal foco de estudo relacionado ao emprego do exercício físico em pacientes em risco ou com DT2, assim como estão bem documentados na literatura os reais benefícios do exercício. Porém, a combinação de duas modalidades de treinamento (aeróbio e resistido) necessita de estudos mais precisos, pois a mesma poderá render melhores resultados do que apenas uma modalidade específica.

Portanto, a proposta deste estudo foi comparar o efeito de duas modalidades de exercício (aeróbio e combinado) no controle glicêmico após 20 semanas de treinamento em pacientes com DT2.

\section{Materiais e métodos}

Tipo de estudo

Esta pesquisa caracteriza-se por ser um estudo quase-experimental, com delineamento longitudinal descritivo, randomizado e prospectivo, tendo uma abordagem quantitativa.

\section{Amostra}

Os participantes do estudo foram compostos por 24 indivíduos portadores de diabetes tipo 2 selecionados a partir do núcleo de apoio da saúde da família do município de Tubarão, Santa Catarina.

Foram selecionados indivíduos que atenderam aos critérios de inclusão: diagnóstico médico de diabetes tipo 2 não insulino-dependente há no mínimo um ano; idade $\geq 40$ anos e $\leq 75$; não estar praticando exercício físico regularmente; e ter atestado médico para a prática de exercício físico.

Os critérios de exclusão foram os seguintes: DM2 insulino-dependente; DM1; DMG; hipertensão descontrolada (PAS > $160 \mathrm{mmHg}$ e PAD > $100 \mathrm{mmHg}$ ); ou pico hipertensivo a menos de um mês do estudo; angina instável e estável; arritmias cardíacas descontroladas; história recente de insuficiência cardíaca congestiva grave (ICC); insuficiência renal e hepática; retinopatia proliferativa aguda; problemas ortopédicos; pé diabético; doenças neurológicas; ser tabagista e/ou etilista.

\section{Procedimentos}

Esta pesquisa seguiu os princípios éticos de respeito à autonomia das pessoas, apontados pela Resolução n. 196, de 10 de outubro de 1996, do Conselho Nacional de Saúde (CNS 196/96). Após apreciação e aprovação pelo Comitê de Ética em Pesquisa (CEP UNISUL), tendo como registro o código 09.071.4.08. III, deu-se início ao programa de treinamento combinado e aeróbio.

Após receberem as informações acerca do estudo, os interessados assinaram o Termo de Consentimento Livre e Esclarecido.

0 grupo foi submetido a coletas referentes à anamnese clínica e às variáveis bioquímicas.

Na primeira sessão de atividades, foi realizada uma anamnese clínica na Clínica Escola de Fisioterapia 
da Unisul (Tubarão, SC). Esta anamnese teve como finalidade triar o paciente e estratificar o seu risco, investigando a presença de acometimento prévio, como doenças cardiovasculares, distúrbios osteomusculares ou de possíveis fatores de risco para a doença arterial coronariana (DAC), administração de fármacos, aplicação do questionário de PARQ 1 e 2 e outras possíveis informações relevantes.

Foram coletadas amostras de sangue dos participantes, após jejum de 10-12 horas, por meio de sistema de coleta a vácuo, em tubos com e sem anticoagulante (EDTA $1 \mathrm{mg} / \mathrm{ml}$ sangue e $\mathrm{NaF}$ ). As amostras de sangue foram centrifugadas a $2.500 \mathrm{rpm}$ por 10 minutos para obtenção do soro ou plasma para serem utilizados nas determinações bioquímicas (glicemia de jejum). As amostras de sangue total, coletadas com EDTA, foram utilizadas para a determinação da hemoglobina glicosilada.

A concentração sérica de glicose foi determinada no sistema Vitros (Johnson \& Johnson). A dosagem de glicose foi determinada paralelamente com soros controle normal (Verify I) e patológico (Verify II).

A hemoglobina glicosilada foi determinada pelo método HPLC (Cromatografia Líquida de Alta Performance) por troca iônica por meio do sistema Bio-Rad Variant II Turbo Hemoglobin Testing System.

Os valores considerados adequados para os parâmetros bioquímicos foram: a glicemia em jejum (GJ) $<100 \mathrm{mg} / \mathrm{dL}^{-1}$ e a hemoglobina glicosilada $<7 \%$ (9-11).

Os participantes foram divididos aleatoriamente em dois grupos de treinamento, sendo o treinamento combinado (TC) e o treinamento aeróbio (TA), com 12 indivíduos em cada grupo.

\section{Treinamento Combinado (TC)}

0 programa de treinamento combinado (resistido e aeróbio) foi constituído de três sessões semanais (segunda, quarta e sexta-feira), durante um período de 20 semanas, totalizando 60 sessões com duração de 60 minutos.

0 TC executado com o grupo foi realizado na sala de musculação da academia ACREF (Associação Cultural e Recreativa dos Funcionários), da Universidade do Sul de Santa Catarina - Câmpus Tubarão. As sessões tiveram início às $8 \mathrm{~h}$ e término às $12 \mathrm{~h}$. 0 grupo foi subdividido em dois horários, das $8 \mathrm{~h}$ às $10 \mathrm{~h}$ e das $10 \mathrm{~h}$ às $12 \mathrm{~h}$, com seis participantes em cada horário, facilitando o atendimento personalizado e promovendo maior atenção aos sujeitos e controle da execução dos exercícios.

Foram realizadas três sessões de exercícios com intervalo de 24 horas para familiarizar os indivíduos com os exercícios utilizados, o ritmo de execução e a definição da técnica de execução. Na segunda e terceira séries, os indivíduos realizavam uma familiarização mais específica para o teste de 1RM, que consistia em realizar de duas a três repetições em cada aparelho, com intervalo de cinco minutos entre as séries e com uma carga próxima a $1 \mathrm{RM}$. A carga foi determinada de forma subjetiva pelo avaliador, pois o objetivo dessa sessão era fazer com que o indivíduo vivenciasse a realização dos exercícios de alta intensidade com o objetivo de que a carga do teste de 1RM fosse a maior possível.

Os sinais vitais (pressão arterial - PA, frequência cardíaca - FC e saturação periférica de oxigênio $\mathrm{SpO}_{2}$ ) foram verificados antes e após as sessões de familiarização.

Com o intuito de reduzir a margem de erro no teste de 1RM, adotou-se a seguinte estratégia:

a) instrução padronizada antes do teste, de modo que o avaliado estivesse ciente de toda a rotina que envolvia a coleta de dados;

b) o avaliado foi instruído sobre a técnica de execução dos exercícios por meio da familiarização com o aparelho e execução do exercício sem carga para reduzir o efeito da fadiga;

c) o avaliador esteve atento quanto à posição adotada pelo praticante, no momento da medida;

d) os testes foram realizados no mesmo horário;

e) estímulos verbais foram realizados a fim de manter alto o nível de estimulação;

f) os indivíduos foram orientados a evitar a manobra de Valsalva.

Para o teste de 1RM, estipulou-se uma carga inicial, utilizando como referência os valores utilizados na fase de familiarização ou a carga considerada pelos sujeitos como o seu valor para 1RM. Em seguida, dava-se um intervalo de descanso de cinco minutos e nova carga era acrescentada para nova execução. 0 teste era concluído quando o participante alcançasse a carga que o levasse à falha mecânica de execução, ficando estabelecida como sua carga máxima a última carga, com a qual conseguiu executar o exercício sem falha mecânica. 
Não foram permitidas mais do que cinco tentativas para o estabelecimento da carga máxima. Caso tal fato ocorresse, o teste era invalidado e o voluntário seria submetido novamente em outro dia. Após a obtenção da carga em um determinado exercício, foram dados intervalos não inferiores a 10 minutos, antes de passar ao teste no exercício seguinte.

As sessões de exercício foram divididas basicamente em seis etapas. Cada um dos participantes teve uma ficha individual para registrar FC, PA, índice de percepção de esforço (IPE), glicemia capilar (antes e depois de cada sessão), série, repetições e carga.

Na primeira etapa (5-10 minutos), que precede o exercício físico, foram realizadas aferições da PA, FC, e IPE.

$\mathrm{Na} 2^{\underline{a}}$ etapa, foram realizados alongamentos para os grandes grupos musculares de 3 a 5 minutos sustentando cada posição por 15 segundos.

$\mathrm{Na} 3{ }^{\mathrm{a}}$ etapa, foi realizado o treinamento aeróbio na esteira. 0 treinamento na esteira seguiu uma velocidade que correspondia a um valor em METs. Inicialmente, permaneciam cinco minutos a uma intensidade de 3 METs, sendo esta aumentada progressivamente até 4 METs, durante 20 minutos. À medida que a velocidade da esteira era aumentada, verificava-se a FC e o IPE dos indivíduos.

$\mathrm{Na} 4^{\mathrm{a}}$ etapa, foi realizado o treino de musculação com aparelhos e/ou pesos livres.

Em relação à ordem de colocação dos exercícios, o treinamento foi dividido em três fases. Na fase I de treinamento (adaptação), com duração de quatro semanas, a distribuição sequencial foi baseada na estratégia alternada de grupamentos musculares. Foram treinados todos os principais grupos musculares em uma mesma sessão.

Nessa fase, foi enfatizada uma intensidade de 40$50 \%$ 1RM, com 3 séries de 12 repetições. Em relação ao método de treinamento de força, foi utilizado o método de esforço submáximo, ou seja, utilização de cargas não máximas com um número intermediário de repetições que não leve à completa exaustão.

Na fase II, da quinta até a décima semana, o programa deixou de ser alternado por segmento e passou a ser localizado por articulação, ou seja, foram colocados em sequência todos os exercícios para o mesmo agrupamento muscular. As variáveis velocidade do movimento, método de treinamento de força, frequência semanal, aparelhos de exercícios e as orientações a respeito da respiração e vestimenta se mantiveram.
Nessa fase do treinamento, foi enfatizada uma intensidade de 50-60\% 1RM, com 3 séries de 12 repetições. Para evitar uma queda no rendimento, os grupos musculares foram divididos em 3 sessões de treino por semana, adotando-se assim, um programa parcelado por segmento.

$\mathrm{Na}$ fase III do treinamento, foi realizado $60-70 \%$ $1 \mathrm{RM}$, com 3 séries de 10 repetições, mantendo o programa parcelado por segmento.

A $5^{\underline{a}}$ etapa foi composta por: resfriamento, aferição dos sinais vitais, identificação do IPE, glicemia capilar.

\section{Treinamento aeróbio (TA)}

Com o grupo de treinamento aeróbio foi feita a caminhada orientada na pista atlética de $198 \mathrm{~m}^{2}$, da Universidade do Sul de Santa Catarina - Câmpus Tubarão. 0 regime de atividades consistiu em 3 sessões semanais de exercício, durante 20 semanas, com início às $18 \mathrm{~h}$ e término às $19 \mathrm{~h}$.

Como método de treinamento, foi empregado o modo contínuo de caminhada, que envolve a aplicação de cargas contínuas caracterizadas pelo predomínio do volume sobre a intensidade, propiciando o desenvolvimento da resistência aeróbia.

No treinamento contínuo, a velocidade da caminhada foi uniforme durante a parte principal do treinamento, respeitando as intensidades determinadas para os participantes, controladas por meio do monitor de frequência cardíaca.

A duração das sessões de treinamento foi de 15 minutos para o paciente iniciante. Essa duração foi sendo aumentada em 5 minutos por semana, até o participante completar os 60 minutos totais da parte aeróbia.

A intensidade da atividade aeróbia foi delimitada por meio da frequência cardíaca alvo, prevista para a idade mediante o método de Karvonen, correspondendo à fase I do treinamento: $40-50 \% \mathrm{FC}$ alvo; Fase II: $50-60 \%$ FC alvo; e Fase III: 60-70\% FC alvo.

As etapas do treinamento aeróbio seguiram o mesmo protocolo do treinamento combinado, ou seja, primeira etapa: aferição dos sinais vitais, identificação do IPE e glicemia capilar; segunda etapa: aquecimento; terceira etapa: treinamento aeróbio; quarta etapa: resfriamento e aferição dos sinais vitais, identificação do IPE e glicemia capilar. 


\section{Análise estatística}

Os dados foram organizados na planilha de cálculo Excel versão Microsoft Windows XP 2002, e analisados no programa Statdisk 11.0.1 for the Triola of Statistic Textbooks, 1986-2009.

Para descrever o perfil da amostra segundo as variáveis em estudo, foi feita uma tabela de frequência das variáveis categóricas (gênero, grupo étnico, medicações), com os valores de frequência relativa (\%). Para as variáveis numéricas, foi realizada estatística descritiva (idade, tempo de diagnóstico) com os valores de média e desvio padrão.

As variáveis glicose em jejum e hemoglobina glicosilada foram submetidas a um teste de normalidade Shapiro Wilk e, assim, foi confirmada uma distribuição gaussiana dos valores.

Para a análise das médias da glicose em jejum e da hemoglobina glicosilada basal e após o programa de treinamento, adotou-se o teste t pareado com nível de significância de $5 \%$ para a análise do estudo.

Os resultados foram apresentados em forma de gráficos, por meio do programa Microcal ${ }^{\mathrm{TM}}$ Origin $^{\mathrm{TM}}$, versão, 5.0 - 1997.

\section{Resultados}

A Tabela 1 apresenta as características dos participantes do treinamento combinado. Dos 12 indivíduos avaliados, $66,67 \%$ são do sexo feminino e $33,33 \%$ do sexo masculino. A média de idade do gênero feminino

Tabela 1 - Características dos participantes no início do estudo do TC $(n=12)$

\begin{tabular}{llc}
\hline Características & & Média \pm ou (\%) \\
\hline Idade em anos & Feminino & $62,62 \pm 5,85$ \\
& Masculino & $65,75 \pm 7,84$ \\
\hline \multirow{2}{*}{ Gênero } & Feminino & 66,67 \\
& Masculino & 33,33 \\
\hline Tempo diabetes & & $12,83 \pm 7,42$ \\
\hline Grupo étnico & Branco & 100 \\
\hline Medicamentos & Monoterapia & 41,67 \\
& Terapia combinada & 58,33 \\
\hline
\end{tabular}

Fonte: Dados da pesquisa. foi de 62,62 \pm 5,85 e masculino de 65,75 \pm 7,84 anos. Quanto à etnia, prevaleceu grupo étnico branco, com $100 \%$. 0 tempo de diagnóstico correspondeu a 12,83 $\pm 7,42$ anos.

Em relação ao perfil dos medicamentos utilizados para o tratamento dos pacientes com diabetes, $58,33 \%$ faziam uso de terapia combinada, ou seja, utilizavam agentes antidiabéticos orais do tipo biguanidas associados a sulfonilureias. Já a monoterapia $33,33 \%$ correspondeu ao uso de sulfonilureias e 8,3\% utilizavam biguanidas.

A Tabela 2 representa as características dos participantes do treinamento aeróbio. Dos 12 indivíduos avaliados, 58,33\% são do sexo feminino e $41,67 \%$ do sexo masculino. A média de idade do gênero feminino foi de 60,14 $\pm 8,39$ e masculino de $53 \pm 76,12$ anos. Quanto à etnia, prevaleceu grupo étnico branco, com $100 \%$. 0 tempo de diagnóstico foi de $11 \pm 5,81$ anos.

Em relação ao perfil dos medicamentos utilizados para o tratamento dos pacientes com diabetes, 50\% faziam uso de terapia combinada, ou seja, utilizavam agentes antidiabéticos orais do tipo biguanidas associados a sulfonilureias. Já a monoterapia correspondeu a $25 \%$ de sulfonilureias e $25 \%$ biguanidas.

0 Gráfico 1 representa o valor da glicose em jejum (mg/dL), basal e após 20 semanas do TC, e do TA. Após 20 semanas de treinamento, houve redução significativa da glicose em jejum do TC (de 167,41 $\pm 38,13$ para $119,83 \pm 20,91$ ) e do TA (de 189,83 \pm 63,57 para $139,91 \pm 34,04$ ) com $\mathrm{p} \leq 0,05$.

No Gráfico 2, está representado o valor da hemoglobina glicosilada (\%), basal e após 20 semanas do

Tabela 2 - Características dos participantes no início do estudo do TA $(n=12)$

\begin{tabular}{llc}
\hline Características & & Média \pm ou (\%) \\
\hline Idade em anos & Feminino & $60,14 \pm 8,39$ \\
& Masculino & $53 \pm 6,12$ \\
\hline \multirow{2}{*}{ Gênero } & Feminino & 58,33 \\
& Masculino & 41,67 \\
\hline Tempo diabetes & & $11 \pm 5,81$ \\
\hline \multirow{2}{*}{ Grupo étnico } & Branco & 100 \\
\hline Medicamentos & Monoterapia & 50 \\
& Terapia combinada & 50 \\
\hline
\end{tabular}

Fonte: Dados da pesquisa. 


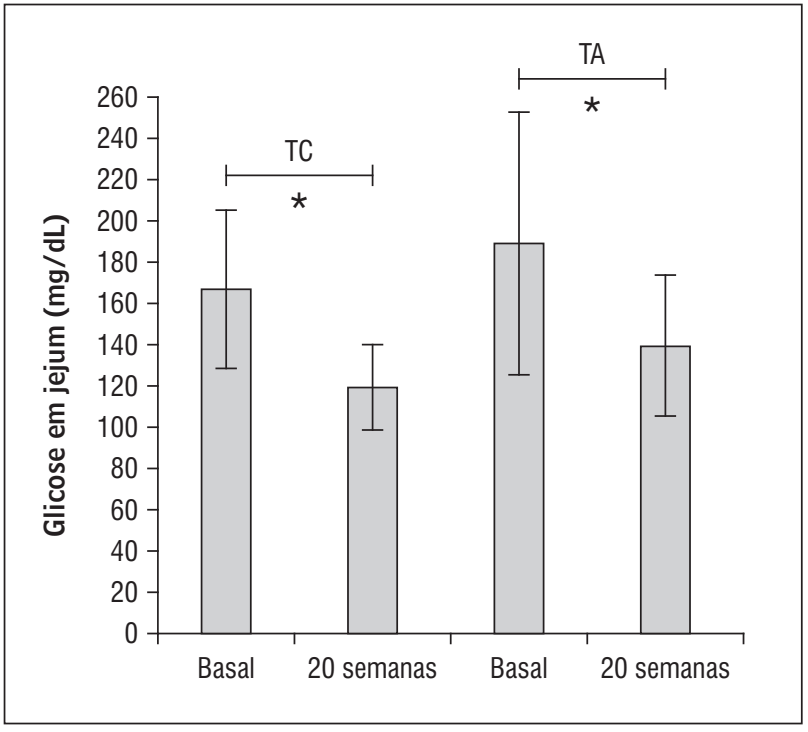

Gráfico 1 - Valores (média e desvio-padrão) da glicose (mg/ $\mathrm{dL}$ ) basal e após 20 semanas de programa de treinamento

Fonte: Dados da pesquisa.

Legenda: $\mathrm{TC}=$ treinamento combinado; $\mathrm{TA}=$ treinamento aeróbio; * $=p<0,05$.

TC, e do TA. Verifica-se que, após 20 semanas de treinamento, houve redução significativa da hemoglobina glicosilada do TC (de 8,61 $\pm 1,17$ para 7,25 $\pm 1,24$ ) e no TA (de $9,52 \pm 2,46$ para $8,37 \pm 1,50$ ) com $\mathrm{p} \leq 0,05$.

\section{Discussão}

Segundo a Sociedade Brasileira de Diabetes (15), o risco de desenvolver diabetes tipo 2 aumenta após os 40 anos de idade, apresentando uma elevação mais aguda na sexta década de vida.

Os resultados do presente estudo corroboram com o estudo desenvolvido por Wild et al. (16), no qual a maioria das pessoas diabéticas encontra-se na faixa etária entre 45 e 64 anos.

Em relação ao uso de medicamentos, o resultado deste estudo vão de encontro ao estudo de Ciechanowiski et al. (17), no qual 52,6\% dos pacientes realizavam tratamento somente com hipoglicemiantes orais, assim como no estudo de Guedes (18) e Faria (19), nos quais a porcentagem de indivíduos diabéticos que faziam uso somente de hipoglicemiantes orais foi de $30,9 \%$ e de $89 \%$, respectivamente.

Atualmente, a biguanida é recomendada como a primeira linha de tratamento e é o sensibilizador de

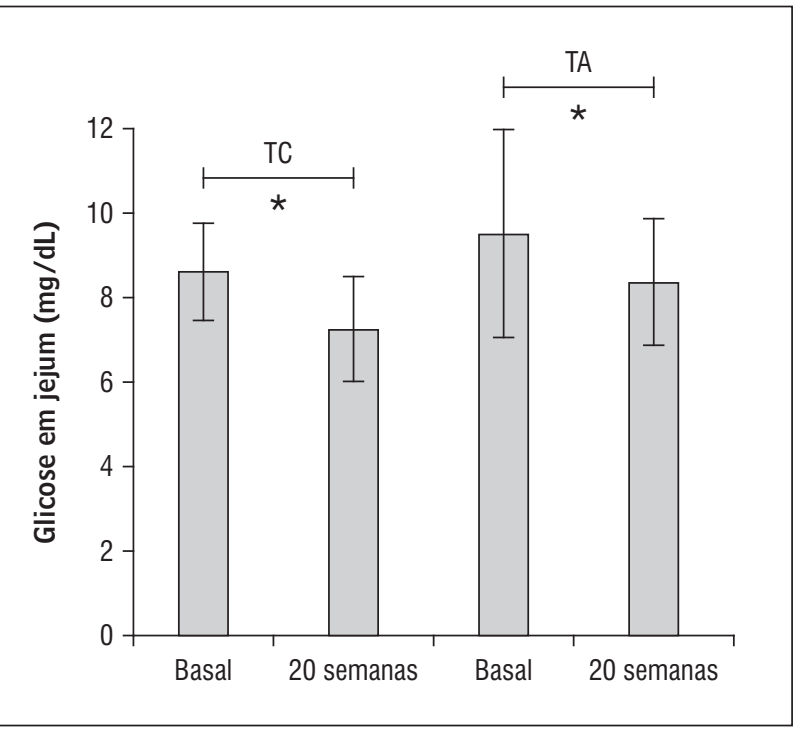

Gráfico 2 - Valores (média e desvio-padrão) da hemoglobina glicada (\%) basal e após 20 semanas de programa de treinamento

Fonte: Dados da pesquisa.

Legenda: $\mathrm{TC}=$ treinamento combinado; $\mathrm{TA}=$ treinamento aeróbio; $\star=p<0,05$.

insulina mais utilizado nos idosos, pois a eficácia na manutenção da glicose no sangue gera baixo risco de hipoglicemia e os efeitos adversos são relativamente baixos (20).

A hipoglicemia é a maior preocupação com a utilização de sulfonilureia, especialmente de ação prolongada, em pacientes idosos, com a função renal reduzida, disfunção hepática e em pacientes com estado nutricional deficiente e uso excessivo de ál$\operatorname{cool}(21,22)$.

No estudo clínico controlado por Dunstan et al. (23), em que foram investigados os efeitos a curto prazo do treinamento resistido sobre o controle glicêmico em pacientes com DT2, foi possível produzir efeito significativo $(\mathrm{p}<0,05)$ sobre o metabolismo de glicose (23).

Já no estudo de Soe et al. (20), os pacientes com DT2 participaram de um circuito de três meses de treinamento de resistência progressiva, apresentando no final da investigação uma melhora significativa $(\mathrm{p} \leq 0,05)$ na hemoglobina glicosilada, corroborando com os achados deste estudo.

A redução nos níveis séricos da HbA1c identificada na presente pesquisa é semelhante àquela observada por Tokmakidis et al. (24), que investigaram os efeitos, a curto e longo prazo, de um programa de 
treinamento combinado no controle glicêmico de 9 mulheres na pós-menopausa. Nele, a hemoglobina glicosilada foi significativamente reduzida após 4 semanas $(\mathrm{p}<0,05)$, e após 16 semanas $(\mathrm{p}<0,01)$.

A análise da hemoglobina glicosilada tem um importante impacto do ponto de vista clínico. Nesse sentido, a dosagem da $\mathrm{HbA} 1 \mathrm{c}$ tem papel fundamental na monitorização do controle glicêmico em pacientes diabéticos, pois fornece informações acerca do índice retrospectivo da glicose plasmática (25-27).

A grande vantagem da HbA1c está no fato de não sofrer grandes flutuações, como na dosagem da glicose plasmática, e estar diretamente relacionada ao risco de complicações em pacientes com diabetes tipo 1 e diabetes tipo 2 (28).

No estudo clínico controlado por Church et al. (29), no qual 262 indivíduos sedentários com diabetes tipo 2 participaram do treinamento combinado, notou-se ganhos significativos nos níveis da HbA1c. 0 grupo de treinamento combinado alcançou redução estatisticamente significativa se comparado ao controle $(0,34 \%)$; já o grupo de treino aeróbio teve uma redução significativa de $0,24 \%$ e o grupo que realizou apenas treinamento resistido obteve uma redução de $0,16 \%$.

Sigal et al. (30) realizaram um estudo randomizado com adultos portadores de DT2 com idades entre 39 e 70 anos, divididos em três grupos (G1: treinamento aeróbio, G2: treinamento resistido e G3: treinamento aeróbio e resistido), com progressão gradual de duração e intensidade. Esses autores obtiveram como resultado um melhor controle glicêmico por meio da redução da hemoglobina glicosilada, alterações no perfil lipídico e composição corporal em todos os participantes, sendo que, no G3, resultou em ganhos maiores. No mesmo estudo, justificam que os efeitos dos treinamentos resistido e aeróbio são complementares. 0 aeróbio tende a aumentar a capacidade cardiorrespiratória, enquanto o treinamento resistido aumenta a força muscular.

Na meta-análise de Selvin et al. (31), constataram que $1 \%$ de decréscimo nos valores da HbA1c está associado a 15-20\% de decréscimo na maioria dos eventos cardiovasculares e $37 \%$ de redução em complicações microvasculares.

Neste estudo, obteve-se uma redução da $\mathrm{HbA1c}$ de $0,16 \%$ no grupo de treinamento combinado e $0,13 \%$ no treinamento aeróbio. Os valores da $\mathrm{HbA1c}$, no começo do programa, também devem ser considerados. A média da HbA1c no início do treinamento combinado foi de 8,6 e no treinamento aeróbio foi de 9,25. Os valores da HbA1c, no TC e no TA estavam distantes (1,6 e 2,5\%, respectivamente) dos níveis de referência.

Segerström et al. (32) examinaram o efeito do treinamento combinado na sensibilidade à insulina e HbA1c em mulheres diabéticas, por um período de seis meses de treino. Os pesquisadores constataram aumento significativo na sensibilidade à insulina e uma significativa diminuição da HbA1c, e concluíram que a sensibilidade à insulina está relacionada diretamente à intensidade do exercício, enquanto que a HbA1c está relacionada principalmente ao volume de treino. 0 presente estudo deu ênfase ao volume de treino ao invés da intensidade, comprovado pela redução mais expressiva da HbA1c no grupo de treinamento combinado.

Marwick et al. (9) concluíram que os estudos anteriores com indivíduos diabéticos que praticaram exercício proporcionaram melhora no controle metabólico, com redução dos níveis da HbA1c na glicose e aumento da sensibilidade à insulina. Porém, na concepção de Brun et al. (33) e Krousel et al. (34), geralmente, os estudos que falharam em obter esses benefícios utilizaram intervenções de baixa intensidade, baixo volume ou baixa adesão à intervenção.

Em outro estudo controlado e randomizado (35), todos os participantes do sexo masculino $(n=20)$ que participaram de exercício resistido ou de exercício aeróbio três vezes por semana durante 10 semanas melhoraram o controle glicêmico. No entanto, a maior redução foi observada no grupo de exercício resistido, no qual após 10 semanas, teve seus níveis de HbA1c significativamente menores do que o grupo que recebeu exercício aeróbio $(\mathrm{p}<0,006)$.

Em uma meta-análise de Snowling e Hopkins (36), após analisar os efeitos de diferentes modos de exercícios, os autores concluíram que todos os tipos de exercícios (aeróbio, resistido e combinado) provocaram reduções da glicemia, sendo que no exercício combinado, obtiveram resultados mais significativos.

Os valores de mudanças da glicose plasmática são similares àqueles encontrados por Maiorana et al. (37), que realizaram um estudo prospectivo e randomizado, para demonstrar que o protocolo de treinamento em circuito, com estações combinadas de treinamento aeróbio e de resistência, melhorou significativamente a glicemia de jejum: de $12.0 \pm 0,5$ para $9.8 \pm 0,5 \mathrm{mmol} \cdot \mathrm{l}^{-1} \mathrm{p}<0,05$. Além disso, os valores da $\mathrm{HbA} 1 \mathrm{c}$ foram significativamente reduzidos: de $8,5 \pm 0,4$ para $7,9 \% \pm 0,3$. 
Por outro lado, outros estudos não apresentaram alteração significativa na concentração de glicose antes e após treinamento aeróbio, como o de Cauza et al. (14), em que relataram uma diminuição significativa de glicose no sangue (de $204 \pm 16 \mathrm{mg} / \mathrm{dL}$ para $147 \pm$ $8 \mathrm{mg} / \mathrm{dL}, \mathrm{p}<0,001$ ) nos participantes de treinamento resistido, enquanto não foram observadas alterações significativas no grupo de treinamento aeróbio.

A principal limitação do estudo foi o tamanho pequeno da amostra, que pode ter limitado o poder do estudo e o tipo de desenho metodológico. Contudo, os resultados são relevantes dentro de uma perspectiva clínica e funcional e vão ao encontro das pesquisas atuais.

\section{Conclusão}

É consenso que o exercício físico deve fazer parte do tratamento do diabetes. Em vista do encontrado, o treinamento físico regular foi capaz de proporcionar mudanças metabólicas que resultaram em melhora da homeostase da glicose. Tanto o treinamento combinado quanto o aeróbio evidenciaram um comportamento positivo no controle glicêmico; porém, o treinamento combinado foi mais eficaz em relação à hemoglobina glicosilada, e o treinamento aeróbio em relação à glicose plasmática.

\section{Referências}

1. Report of expert committee on the diagnosis and classification of diabetes mellitus. Diabetes Care. 1997; 20(7):1183-97.

2. Sociedade Brasileira de Diabetes - SBD. Diagnóstico e tratamento do diabetes mellitus e tratamento do diabetes mellitus tipo 2: recomendações da Sociedade Brasileira de Diabetes [Internet] 2002 [acesso 6 mar. 2011]. Disponível em: http://www.diabetes.org.br.

3. Sociedade Portuguesa de Diabetes - SPD. Definição, diagnóstico e classificação da diabetes mellitus. [Internet] 1999 [acesso 6 mar. 2011]. Disponível em: http://www.spd.pt/classif.html.

4. World Health Organization. Global strategy on diet, physical activity and health. WHO [Internet] 2003 [acesso 6 mar. 2011]. Disponível em: http://www. who.int/hpr/NPH/docs/gs_diabetes.pdf.
5. Organização Pan-Americana da Saúde, Organização Mundial da Saúde. Doenças crônico-degenerativas e obesidade: estratégia mundial sobre alimentação saudável, atividade física e saúde. 2003. [acesso 6 mar. 2011]. Disponível em: http://www.opas.org.br/sistema/arquivos/d_cronic.pdf.

6. Brasil. Ministério da Saúde e Secretaria de Políticas de Saúde Plano de Reorganização da Atenção à Hipertensão arterial e ao Diabetes mellitus. Manual de Hipertensão arterial e Diabetes mellitus. Brasília: Ministério da Saúde; 2002.

7. Hordern MD, Dunstan DW, Prins JB, Baker MK, Singh MA, Coombes JS. Exercise prescription for patients with type 2 diabetes and pre-diabetes: a position statement from exercise and sport science Australia. J Sci Med Sport. 2012;15(1):25-31.

8. Association Diabetes American: standards of medical care in diabetes - 2009. Diabetes Care. 2009;32 Suppl 1:S13-61.

9. Marwick TH, Hordern MD, Miller T, Chyun DA, Bertoni AG, Blumenthal RS, et al. Exercise training for type 2 diabetes mellitus impact on cardiovascular risk: a scientific statement from the American Heart Association. Circulation. 2009;119(25):3244-62.

10. Praet $S$, van Loon L. Exercise therapy in type 2 diabetes. Acta Diabetol. 2009;46(4):263-78.

11. Zanuso S, Jimenez A, Pugliese G, Corigliano G, Balducci S. Exercise for the management of type 2 diabetes: a review of the evidence. Acta Diabetol. 2010;47(1): 15-22.

12. O'Donovan G, Kearney E, Nevill A, Woolf-May K, Bird $\mathrm{S}$. The effects of 24 weeks of moderate- or high-intensity exercise on insulin resistance. Eur J Appl Physiol. 2005;95(5-6):522-8.

13. Hordern M, Coombes J, Cooney L, Jeffriess L, Prins J, Marwick T. Effects of exercise intervention on myocardial function in type 2 diabetes. Heart. 2009;95(16): 1343-9.

14. Cauza E, Hanusch-Enserer U, Strasser B, Ludvik B, Metz-Schimmerl S, Pacini G, et al. The relative benefits of endurance and strength training on the metabolic factors and muscle function of people with type 2 diabetes mellitus. Arch Phys Med Rehabil. 2005;86(8): 1527-33. 
15. Sociedade Brasileira de Diabetes (SBD). Diretrizes da Sociedade Brasileira de Diabetes. SBD 2008. [acesso 20 jun. 2010];1-108. Disponível em: http://www. diabetes.org.br/educacao/docs/Diretrizes_SBD_2008_ MAR_12.pdf.

16. Wild S, Roglic G, Green A, Sicree R, King H. Global prevalence of diabetes: estimates for the year 2000 and projections for 2030. Diabetes Care. 2004;27(5):1047-53.

17. Ciechanowiski PS, Katon WJ, Russo JE, Walker EA. The patient-provider relationship: attachment theory and adherence to treatment in diabetes. Am J Psychiatry. 2001 [acesso 19 jan. 2011];158(1):29-35. Disponível em: http://ajp.psychiatryonline.org/cgi/ reprint/158/1/29.

18. Guedes AC. A associação entre o perfil clínico e psicossocial de pessoas com diabetes mellitus usuárias de uma unidade de saúde da família de Sorocaba - SP. (dissertação). São Paulo: Escola de Enfermagem da Universidade de São Paulo; 2007.

19. Faria HTG. Fatores relacionados à adesão do paciente diabético à terapêutica medicamentosa. (dissertação). São Paulo: Escola de Enfermagem de Ribeirão Preto da Universidade de São Paulo. [acesso 8 ago. 2010]. Disponível em: http://www.teses.usp.br/teses/ disponiveis/22/22132/tde-12032008-100144/ $? \mathrm{C}=\mathrm{N} ; \mathrm{O}=\mathrm{D}$.

20. Soe K, Sacerdote A, Karam J, Bahtiyar G. Management of type 2 diabetes mellitus in the elderly. Maturitas. 2011;70(2):151-9.

21. DeFronzo RA. Pharmacologic therapy for type 2 diabetes mellitus. Ann Intern Med. 1999;131(4):281-303.

22. Inzucchi SE. Oral antihyperglycemic therapy for type 2 diabetes: scientific review. JAMA. 2002;287(3): 360-72.

23. Dunstan DW, Puddey IB, Beilin LJ, Burke V, Morton AR, Stanton KG. Effects of a short-term circuit weight training program on glycemic control in NIDDM. Diabetes Res Clin Pract. 1998;40(1):53-61.

24. Tokmakidis SP, Zois CE, Volaklis KA, Kotsa K, Touvra AM. The effects of a combined strength and aerobic exercise program on glucose control and insulin action in women with type 2 diabetes. Eur J Appl Physio. 2004 [acesso 26 set. 2010];(92)4-5:437-442. Disponível em: http://www.springerlink.com/content/ aq81mpwcnyyy8q00/fulltext.pdf?page=1.
25. Bry L, Chen PC, Sacks DB. Effects of hemoglobin variants and chemically modified derivatives on assays for glycohemoglobin. Clin Chem. 2001;47(2):153-63.

26. Sacks DB, Bruns DE, Goldstein DE, Maclaren NK, McDonald JM, Parrott M. Guidelines and recomendations for laboratory analysis in the diagnosis and management of diabetes mellitus. Clin Chem. 2002;48(3):436-72.

27. UK Prospective Diabetes Study. Intensive blood-glucose control with sulphonylureas or insulin compared with conventional treatment and risk of complications in patients with type 2 diabetes. Lancet. 1998; 352(9131):837-53.

28. Diabetes Control and Complications Trial - DCCT. The effect of intensive treatment of diabetes on the development and progression of the long-term complications in insulin-dependent diabetes mellitus. N Engl J Med. 1993;329(14):977-86.

29. Church TS, Blair SN, Cocreham S, Johannsen N, Johnson W, Kramer K, et al. Effects of aerobic and resistance training on hemoglobin A1c levels in patients with type 2 diabetes: a randomized controlled trial. JAMA. 2010;304(20):2253-62.

30. Sigal RJ, Kenny GP, Boule NG, Wells GA, Prud'homme D, Fortier M, et al. Effects of aerobic training, resistance training, or both on glycemic control in type 2 diabetes: a randomized trial. Ann Intern Med. 2007; 147(6):357-69.

31. Selvin E, Marinopoulos S, Berkenblit G, Rarni T, Brancati FL, Powe NR, et al. Metanalysis: glycosylated hemoglobin and cardiovascular disease in diabetes mellitus. Ann Intern Med. 2004;141(6):421-31.

32. Segerström AB, Glans F, Eriksson KF, Holmbäck AM, Groop L, Thorsson 0, et al. Impact of exercise intensity and duration on insulin sensitivity in women with T2D. Eur J Intern Med. 2010;21(5):404-8.

33. Brun JF, Bordenave S, Mercier J, Jaussent A, Picot MC, Préfaut C. Cost-sparing effect of twice-weekly targeted endurance training in type 2 diabetics: a one-year controlled randomized trial. Diabetes Metab. 2008; 34(3):258-65.

34. Krousel-Wood MA, Berger L, Jiang X, Blonde L, Myers L, Webber L. Does home-based exercise improve body mass index in patients with type 2 diabetes? Results of a feasibility trial. Diabetes Res Clin Pract. 2008; 79(2):230-6. 
35. Bweir S, Al-Jarrah M, Almalty AM, Maayah M, Smirnova IV, Novikova L, et al. Resistance exercise training lowers HbA1c more than aerobic training in adults with type 2 diabetes. Diabetol Metab Syndr. 2009;1:27.

36. Snowling NJ, Hopkins WG. Effects of different modes of exercise training on glucose control and risk factors for complications in type 2 diabetic Patients. Diabetes Care. 2006;29(11):2518-27.
37. Maiorana A, O’Driscoll G, Cheetham C, Dembo L, Stanton $\mathrm{K}$, Goodman C, et al. The effect of combined aerobic and resistance exercise training on vascular function in type 2 diabetes. J Am Coll Cardiol. 2001;38(3): 860-6.

Recebido: 07/02/2011

Received: 02/07/2011

Aprovado: 01/08/2011

Approved: 08/01/2011 